\title{
MicroRNA-17 downregulates expression of the PTEN gene to promote the occurrence and development of adenomyosis
}

\author{
HAIYAN HU' ${ }^{1}$, HUIJUAN $\mathrm{LI}^{2}$ and YUANLI HE${ }^{2}$ \\ ${ }^{1}$ Department of Obstetrics and Gynecology, Shenzhen Meternal and Child Healthcare Hospital, Southern Medical University, \\ Guangzhou, Guangdong 518028; ${ }^{2}$ Department of Obstetrics and Gynecology, Zhujiang Hospital, \\ Southern Medical University, Guangzhou, Guangdong 510282, P.R. China
}

Received November 17, 2015; Accepted March 10, 2017

DOI: $10.3892 /$ etm.2017.5013

\begin{abstract}
The aim of the current study was to evaluate the expression of microRNA (miR)-17 in the endometrial tissues of patients with adenomyosis (AM) and determine its biological function in the occurrence and development of the disease. A total of 45 fresh endometrial tissues of AM patients and 32 normal endometrial tissues were collected from healthy controls. The expression of miR-17 was evaluated using reverse transcription-quantitative polymerase chain reaction (RT-qPCR). The miR-17-targeting gene phosphatase and tensin homolog (PTEN) was predicted using bioinformatics and its expression was evaluated with RT-qPCR and western blot analysis. Endometrial cells were isolated from patients with AM and healthy controls. They were cultured in vitro and transfected with antagomiR-17 to downregulate miR-17 expression, subsequently cell viability and apoptosis were measured using MTT and flow cytometry. The expression of PTEN and cell cycle- and apoptosis-related proteins were evaluated using western blot analysis. Endometrial cells that stably overexpressed PTEN were screened in vitro by co-culture with G418. A dual-luciferase reporter assay was conducted to verify whether miR-17 was directly bound to PTEN mRNA. The results demonstrated that expression of miR-17 was significantly increased in the endometrial tissues of patients with AM compared with control patients $(\mathrm{P}<0.05)$. PTEN mRNA and protein expression were significantly lower in the AM group compared with the control group $(\mathrm{P}<0.05)$. When the expression of miR-17 in the cells was downregulated, the expression of PTEN was significantly increased $(\mathrm{P}<0.05)$. In addition, expression of $\mathrm{Bcl}-2$ protein was significantly decreased and that of Bax protein significantly increased compared with the negative control (both $\mathrm{P}<0.05$ ). The expression of cyclins E1
\end{abstract}

Correspondence to: Dr Yuanli He, Department of Obstetrics and Gynecology, Zhujiang Hospital, Southern Medical University, 253 Gongye Road, Guangzhou, Guangdong 510282, P.R. China E-mail: heyuanli310@163.com

Key words: microRNA-17, adenomyosis, phosphatase and tensin homolog and D1 were also significantly downregulated $(\mathrm{P}<0.05)$. When PTEN was overexpressed or miR-17 was downregulated, the viability of endometrial cells significantly decreased and cell apoptosis significantly increased (all $\mathrm{P}<0.05$ ). A dual-luciferase reporter assay indicated that miR-17 could directly bind to the PTEN mRNA 3'-untranslated region to regulate its expression. Thus the current study indicates that expression of miR-17 was increased in the endometrial tissues of patients with AM and may influence cell apoptosis and cyclin expression through the targeted regulation of PTEN. These results suggest that miR-17 promotes the occurrence and development of AM.

\section{Introduction}

Adenomyosis (AM) is a pathological change caused by invasion of the endometrium, which normally lines the uterus, into the myometrium (1), which is accompanied by compensatory hyperplasia of the surrounding muscle cells. When lesions in the myometrium exhibit limited nodules, the condition is also referred to as adenomyoma. The etiology and molecular mechanisms of AM remain unclear (2). AM usually occurs in mothers $>40$ years old, with an incidence of $15-70 \%$ among this population sub-group (3). The primary clinical manifestations of patients are excessive menstrual bleeding, menstrual extension, dysmenorrhea, dyspareunia and chronic pelvic pain. The incidence of dysmenorrhea in patients with AM is $>70 \%$ (4). Thus, the health and daily activities of patients with severe AM may be greatly affected by the disease. Recent research has indicated that the incidence of AM is closely associated with ovarian hormone secretion $(5,6)$. Under the influence of ovarian hormones, ectopic endometrial tissues undergo periodical changes and induce pathological changes of the metastatic sites, resulting in chronic pelvic pain and infertility $(7,8)$. Therefore, studies investigating the molecular mechanism of the occurrence and development of AM are clinically significant.

MicroRNAs (miRs) are a key post-transcriptional factor. They are small RNA molecules of 18-22 nucleotides in length that specifically bind to the 3'-untranslated region (UTR) of mRNA to inhibit protein translation (9). Previous studies have determined that miRs serve important roles in tumors and various diseases, including cardiovascular disease and endocrine disease $(10,11)$. Furthermore, miR-17 has been reported to serve important roles in the processes of tumor proliferation, invasion, 
metastasis and apoptosis $(12,13)$. However, there have been few studies investigating the roles of miRNA in the occurrence and development of AM. Although AM is a benign lesion, it exhibits similar biological behaviors to malignant tumor cells, namely invasion and distant metastasis (14). Therefore, it is speculated that miR-17, which serves important roles in the occurrence and development of many types of tumors (15), also participates in the occurrence and development of AM. In the current study, the expression of miR-17 in the endometrial tissues of AM patients was analyzed using reverse transcription-quantitative polymerase chain reaction (RT-qPCR). Expression of PTEN, apoptosis- and cell-cycle-related proteins were detected by immunohistochemistry and western blotting, and their association with miR-17 expression was analyzed. The biological function of miR-17 and associated molecular mechanisms were studied in primary endometrial tissue cells, with the aim of identifying novel targets for the diagnosis and treatment of AM.

\section{Materials and methods}

Collection of tissue samples. A total of 45 endometrial tissues were surgically collected from 45 patients clinically and pathologically diagnosed with AM between January 2013 and April 2014 in the Department of Obstetrics and Gynecology, Zhujiang Hospital (Guangzhou, China). The patients were 21-49 years old (mean, 44.5 years; median, 41 years). The inclusion criteria were as follows: No preoperative chemotherapy, no chronic basic disease and no long history of medication. A further 32 endometrial tissues were collected from 32 patients who underwent hysteroscopy surgery due to abnormal uterine mediastinum and used as normal controls (mean age, 35.6 years). Normal controls were not suffering from any other diseases. No patients received any hormone therapy 3 months prior to surgery and no serious complications arose during surgery. Prior written and informed consent was obtained from all participants and the current study was approved by the ethics review board of Southern Medical University (Guangzhou, China).

Cell culture. Endometrial stromal cells (ESCs) were cultured according to the method reported by Mei et al (16). Briefly, endometrial tissues were cut into small pieces and digested at $37^{\circ} \mathrm{C}$ for $\sim 70 \mathrm{~min}$ and pipetted for $20 \mathrm{~min}$ to disperse the cells. Digested tissues were filtered and the filtrate was centrifuged at 1,000 $\mathrm{x}$ g for $5 \mathrm{~min}$ at room temperature. ESCs were seeded onto culture plates at a concentration of $10^{5}$ cells $/ \mathrm{ml}$ and allowed to adhere for $60 \mathrm{~min}$. The cells were washed twice with PBS and then transferred into Dulbecco's modified Eagle's medium (DMEM)/F12K medium (BD Biosciences, Franklin Lakes, NJ, USA) supplemented with $10 \%$ fetal bovine serum (FBS, BD Biosciences). 293T cells (Cell Bank of Chinese Academy of Sciences, Shanghai, China) were cultured in RPMI-1640 medium (BD Biosciences) supplemented with 10\% FBS. Both cell types were cultured at $37^{\circ} \mathrm{C}$ under a humid atmosphere with $5 \% \mathrm{CO}_{2}$.

Reagents. TRIzol for the extraction of total RNA from tissue cells was purchased from Invitrogen (Thermo Fisher Scientific, Inc., Waltham, MA, USA). The Takara PrimeScript RT Reagent kit and SYBR PrimeScript RT-PCR Reagent kit were purchased from Takara Biotechnology Co., Ltd. (Dalian, China). Rabbit anti-human PTEN polyclonal antibody (cat. no. ab31392) was obtained from Abcam (Cambridge, MA, USA). The FITC Annexin V Apoptosis Detection kit I was purchased from BD Biosciences and the dual-luciferase reporter assay system was purchased from Promega Corporation (Madison, WI, USA).

$R T-q P C R$. Endometrial tissues were ground in liquid nitrogen and $1 \mathrm{ml} / 100 \mathrm{mg}$ TRIzol was added for full lysis. Total RNA was extracted using the phenol chloroform method (17) and the integrity of RNA strands was assessed using gel electrophoresis. The purity of the RNA was measured using a spectrophotometer (Nanodrop ND1000; Thermo Fisher Scientific Inc.) for the 260/280 ratio. Then, $1 \mu \mathrm{g}$ total RNA underwent reverse transcription using the Takara PrimeScript RT Reagent kit, according to the manufacturer's instructions. The template cDNA was stored at $-20^{\circ} \mathrm{C}$. The reaction system of RT-qPCR was as follows: $10 \mu \mathrm{l}$ RT-qPCR-Mix, $5 \mu \mathrm{l}$ upstream primer, $5 \mu \mathrm{l}$ downstream universal primers, $2 \mu \mathrm{l} \mathrm{cDNA}$ and $7 \mu \mathrm{ldd} \mathrm{H}_{2} \mathrm{O}$. The reaction program was as follows: Pre-denaturation at $95^{\circ} \mathrm{C}$ for $10 \mathrm{~min}$ followed by 40 cycles of $95^{\circ} \mathrm{C}$ for $1 \mathrm{~min}$ and $6^{\circ} \mathrm{C}$ for $30 \mathrm{sec}$. The results were quantified using the $2^{-\Delta \Delta \mathrm{Cq}}$ method, and normalized to the level of GADPH (18). The experiment was repeated three times.

Western blot analysis. The endometrial tissues were ground in liquid nitrogen, then radioimmunoprecipitation (RIPA) lysis buffer containing $1 \%$ phenylmethane sulfonyl fluoride and protease inhibitors (Beyotime Institute of Biotechnology, Beijing, China) was added and mixed well by pipetting. Lysed tissues were stored at $4^{\circ} \mathrm{C}$ overnight and subsequently centrifuged at $12,000 \mathrm{x} \mathrm{g}$ for $10 \mathrm{~min}$ at $4^{\circ} \mathrm{C}$. The supernatant was aliquoted and added to $5 \mathrm{x}$ loading buffer prior to electrophoresis. Following mixing, the sample was denatured in a boiling water bath for $10 \mathrm{~min}$.

For the cell samples $\left(2 \times 10^{5}\right.$ cells/well $)$, the processing method was as follows: $200 \mu 1$ RIPA lysis buffer and protease inhibitors were added to each well of the 24-well plate and placed on ice for $5 \mathrm{~min}$, then $5 \mathrm{x}$ loading buffer was added and mixed well, then the samples were denatured in a boiling water bath for $10 \mathrm{~min}$.

For each sample, $10 \mu 1$ protein was loaded per lane prior to $12 \%$ SDS-PAGE. Protein bands were transferred to a PVDF membrane at a constant current of $300 \mathrm{~mA}$ for $1 \mathrm{~h}$ and blocked with $50 \mathrm{~g} / 1$ skim milk at room temperature for $1 \mathrm{~h}$. An appropriate concentration of primary antibody (PTEN 1:800, ab31392; Bcl-2 1:800, ab59348; Bax 1:800, ab53154; cyclin D1 1:800, ab61758; cyclin E1 1:800, ab88259; GAPDH 1:5,000, ab8245) was added and incubated at $4^{\circ} \mathrm{C}$ overnight on a shaker. The next day, membranes were washed with PBS with Tween-20 (PBST) five times for 5 min each time, then horseradish peroxidase-labeled secondary antibodies (goat anti-mouse 1:3,000, ab6789; goat anti-rabbit 1:3,000 ab6721) were added and incubated for $1 \mathrm{~h}$ at room temperature. The membrane was then washed with PBST five times for $5 \mathrm{~min}$ each time and developed using an enhanced chemiluminescence kit (Hanheng Biotechnology, Shanghai, China). The grey values of the protein bands were quantified with Quantity One 4.1 software (Bio-Rad Laboratories, Inc., Hercules, CA, USA) and the results were the average of three independent experiments. 
Transfection of ESCs with antagomiR-17. ESCs at logarithmic growth phase were seeded in a 24-well plate at a concentration of $3 \times 10^{5}$ cells/well the day prior to transfection. Cells were cultured in antibiotic-free F12/DMEM medium containing $10 \%$ FBS. Cell adhesion and growth were observed under an optical microscope on the next day and transfected upon reaching $70 \%$ confluence. A mixture consisting of $1.5 \mu \mathrm{l}$ antagomiR-17 (25 nM; Hanheng Biotechnology), $1 \mu$ l Lipofectamine 2000 (Invitrogen; Thermo Fisher Scientific, Inc.) and $50 \mu \mathrm{l}$ Opti Memi medium (Thermo Fisher Scientific, Inc.) was prepared in an Eppendorf tube and added to the wells after they had been standing for $20 \mathrm{~min}$ at room temperature. Following $6 \mathrm{~h}$ culture at $37^{\circ} \mathrm{C}$, the medium was replaced with fresh F12/DMEM medium containing $10 \% \mathrm{FBS}$ and cultured at $37^{\circ} \mathrm{C}$ for a further $48 \mathrm{~h}$. Untreated cells were used as the blank control, while cells transfected with empty vectors were used as the negative control. The cell proteins were collected and the expression of the target PTEN protein, apoptosis and cell cycle-related proteins were evaluated by western blot analysis, as described above.

MTT assay. Three groups of ESC cells, including control, negative control and antagomiR-17, were seeded in a 96-well plate at a concentration of $2 \times 10^{3}$ cells/well, and each measurement was measured in triplicate. Following 24, 48 and $72 \mathrm{~h}$ culture, $20 \mu \mathrm{l}$ of $5 \mathrm{~g} / \mathrm{l} \mathrm{MTT}$ was added to the wells and incubated for $4 \mathrm{~h}$ at $37^{\circ} \mathrm{C}$. Subsequently, $150 \mu \mathrm{l}$ dimethyl sulfoxide was added to each well to dissolve MTT. The absorbance at $490 \mathrm{~nm}$ for each well was measured with a plate reader and cell viability curves were plotted. Untreated cells were used as the blank control, while cells transfected with empty vectors were used as the negative control.

Detection of cell apoptosis. Each group of transfected cells was collected by $0.25 \%$ trypsin digestion, centrifuged at $800 \mathrm{x}$ g for $5 \mathrm{~min}$ at room temperature and washed twice with pre-cooled PBS. Cells were treated according to the manufacturer's instructions using the FITC Annexin V Apoptosis Detection kit I and cell apoptosis in each group was evaluated by BD FACSVerse ${ }^{\mathrm{TM}}$ flow cytometry (BD Biosciences, US). Annexin V positive alone was considered to indicate early apoptosis, propidium iodide (PI) positive alone was considered to indicate necrosis, and both Annexin V and PI positive were recognized to be indicative of late apoptosis. The results were analyzed by ModFit 2.3 software (Verity Software House, Inc., Topsham, ME, USA).

Prediction of miR-17 target genes. The online Targetscan software (version 7.1, www.targetscan.com) was used for target gene prediction.

Screening of PTEN overexpression cells. The PTEN overexpression plasmid GV142-PTEN (Hanheng Biotechnology) was transfected into the endometrialcells using Lipofectamine ${ }^{\circledR} 2000$. The cells were incubated at $37^{\circ} \mathrm{C}$ in complete DMEM/F12

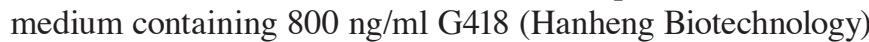
for 7 days for selection of positive cells, then incubated for a further 14 days in complete medium containing $400 \mathrm{ng} / \mathrm{ml} \mathrm{G} 418$. Cells were then seeded in a 96-well plate $(3,000$ cells/well) using the limiting dilution method (19) and cultured at $37^{\circ} \mathrm{C}$ in DMEM/F12 medium containing $200 \mathrm{ng} / \mathrm{ml} \mathrm{G418.} \mathrm{The} \mathrm{trans-}$ fection efficiency and purity of positive cells were evaluated according to the presence of green fluorescence. Positive clones were selected and expanded to detect changes in cell viability and apoptosis.

Dual-luciferase reporter assay. The miR-17 normal and mutated seed sequence in the 3'-UTR of the PTEN gene were chemically synthesized in vitro (Hanheng Biotechnology), and the restriction sites of Spe1 and HindIII were added to both ends, respectively. The two DNA fragments were cloned into a pMIR-REPORT luciferase reporter plasmid and the mutated 3'-UTR seed sequence was used as the control. The plasmids containing 3'-UTR and mutated 3'-UTR sequences were transfected into 293T cells using the liposome method (20). miR-17 mimics (Hanheng Biotechnology) were transfected and cultured at $37^{\circ} \mathrm{C}$ for $24 \mathrm{~h}$, then cells from each group were lysed and fluorescence was measured with a GloMax 20/20 luminometer (Promega Corporation) using Renilla luciferase as the internal standard. All procedures were operated strictly according to the manufacturer's instructions for the dual-luciferase reporter assay kit.

Statistical analysis. All data are expressed as the mean \pm standard deviation. Data were processed using SPSS 11.0 statistical software (SPSS, Inc., Chicago, IL, USA). Student's t-test was used to compare the groups and $\mathrm{P}<0.05$ was considered to indicate a statistically significant difference.

\section{Results}

Expression of miR-17 in the endometrial tissues of AM. To determine the expression of miR-17 in AM tissues, RT-qPCR was performed. The results indicated that expression of miR-17 in the endometrial tissues of AM was 4.82 \pm 0.27 , which was significantly higher compared with the control group $(\mathrm{P}<0.05$; Fig. 1A). Levels of PTEN mRNA and protein in the AM tissues were $0.42 \pm 0.07$ and $0.51 \pm 0.10$, respectively, which were significantly lower compared with the control levels (both $\mathrm{P}<0.05$; Fig. 1A-C). This suggests that miR-17 in the endometrial tissues of AM is associated with decreased expression of PTEN.

Effect of miR-17 on the viability of ESCs. To determine the effect of miR-17 on ESC viability, an MTT assay was conducted. It was demonstrated by western blot analysis that the expression of miR-17 in the endometrial cells was significantly downregulated following transfection with antagomiR-17 compared with controls $(\mathrm{P}<0.05$; Fig. $2 \mathrm{~A})$. The results of the MTT assay indicated that cell viability significantly decreased following downregulation of miR-17 by antagomiR-17 compared with controls $(\mathrm{P}<0.05$; Fig. 2B). This result suggests that miR-17 may promote the viability of ESCs.

Effect of miR-17 on apoptosis of ESCs. To determine whether miR-17 affected ESC apoptosis, flow cytometry was performed to determine the apoptotic rate of ESCs following downregulation of miR-17 by antagomiR-17. The results indicated that the apoptotic rate of ESCs increased significantly following downregulation of miR-17 ( $\mathrm{P}<0.05$; Fig. 3). This suggests that inhibition of miR-17 in the endometrial tissues of AM patients may induce apoptosis of endometrial cells and thus inhibit their metastasis. 
A

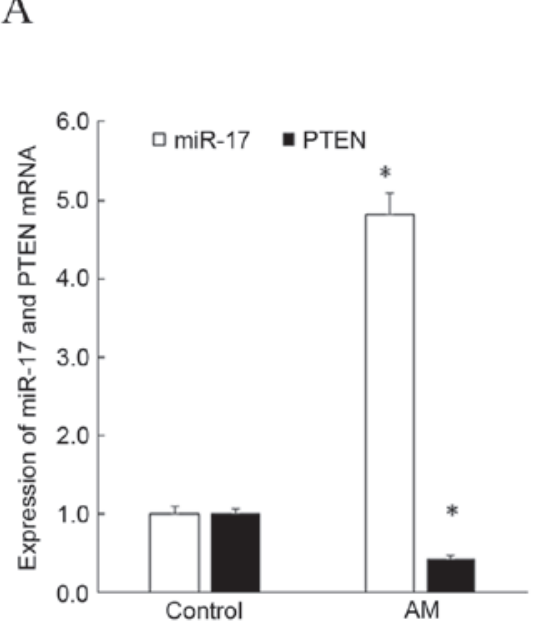

B

Control

AM

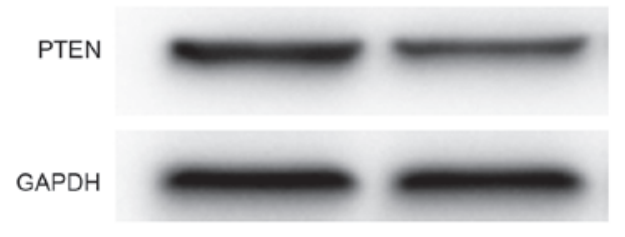

$\mathrm{C}$

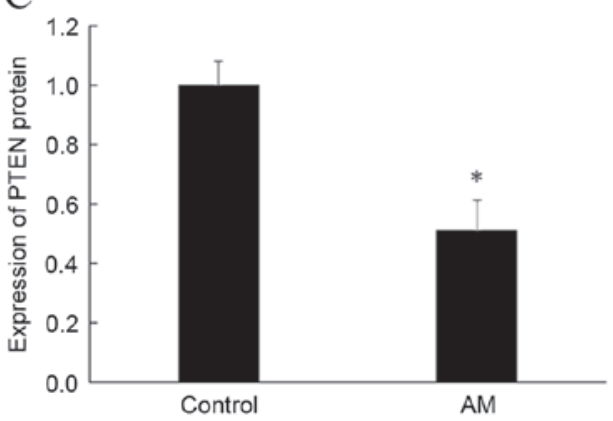

Figure 1. Expression of miR-17 and PTEN in the endometrial tissues of AM patients. (A) Expression of miR-17 and PTEN mRNA in AM and control tissues. (B) Western blot analysis and (C) quantification of PTEN protein expression in AM and control tissues. The mRNA and protein levels were normalized to GADPH. "P<0.05 vs. control group. AM, adenomyosis; miR-17, microRNA-17; PTEN, phosphatase and tensin homolog.
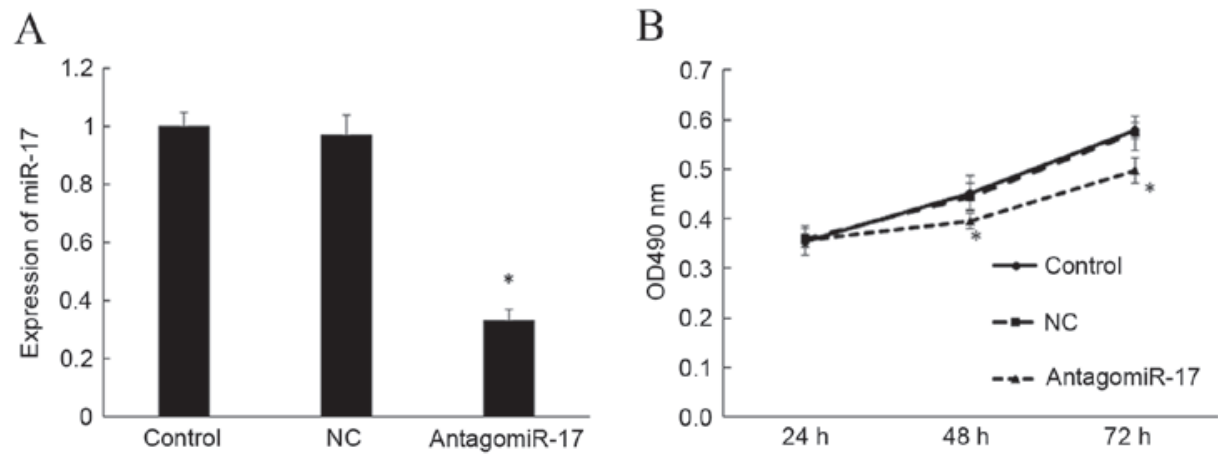

Figure 2. Effect of miR-17 on the viability of endometrial cells. (A) Expression of miR-17 following transfection with antagomiR-17. The miRNA levels were normalized to GADPH. (B) Cell viability following downregulation of miR-17 with antagomiR-17, evaluated using an MTT assay. ${ }^{*} \mathrm{P}<0.05$ vs. control group. miR-17, microRNA-17; NC, negative control; OD, optical density.

Effect of miR-17 on the expression of PTEN and related proteins. To further study the molecular biological mechanism of miR-17, the expression of its potential target gene PTEN was evaluated, as well as the expression of the apoptosis-related proteins BAX and $\mathrm{Bcl}-2$, and the cell cycle-related proteins, cyclins D1 and E1. When the expression of miR-17 in ESCs was downregulated, the expression of PTEN protein was significantly increased compared with the negative control group $(\mathrm{P}<0.05$; Fig. 4). No significant differences were found between the control and negative control groups. When miR-17 was downregulated, the expression of $\mathrm{Bcl}-2$ protein, cyclins D1 and E1 significantly declined, whereas expression of Bax significantly increased compared with the negative control group (all $\mathrm{P}<0.05$; Fig. 4). There were no significant differences in the expression of these proteins between the control and negative control groups. These results suggest that miR-17 is closely associated with the cell cycle and apoptosis of endometrial cells.

Effect of PTEN overexpression on the viability and apoptosis of ESCs. To confirm that miR-17 exerts its biological activity through regulation of PTEN, the effects of PTEN on the viability and apoptosis of ESCs were investigated. Endometrial cells that stably overexpressed PTEN and could stably display green fluorescence, was successfully screened (Fig. 5A). When PTEN was overexpressed, the viability of ESCs significantly decreased $(\mathrm{P}<0.05$; Fig. 5B) and apoptosis significantly increased $(\mathrm{P}<0.05$; Fig. 5C) compared with the control group. There were no significant differences between the control and negative control groups. These results were similar to the effects of miR-17, further suggesting that miR-17 may exert its biological function by regulating PTEN.

MiR-17 may bind to the 3'-UTR of PTEN mRNA. To demonstrate that miR-17 directly binds to the 3'-UTR of PTEN mRNA, a dual-luciferase reporter assay was performed. The results indicated that co-transfection of miR-17 mimics and pMIR-REPORT luciferase reporter plasmid resulted in a significant decrease in fluorescence $(\mathrm{P}<0.05$; Fig. 6), indicating a decrease in PTEN expression. There was no statistical difference between the mutant 3'-UTR group and control group. 


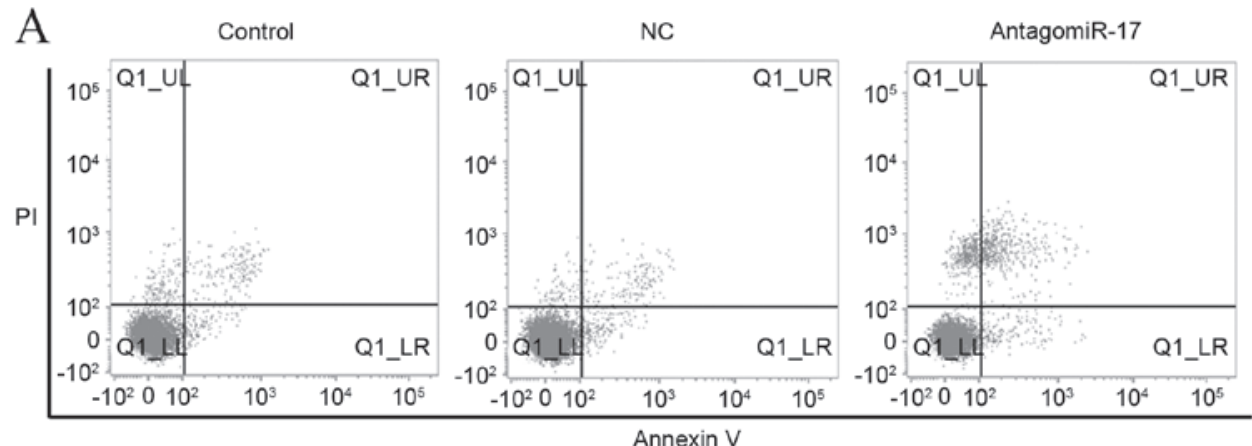

$\mathrm{B}$

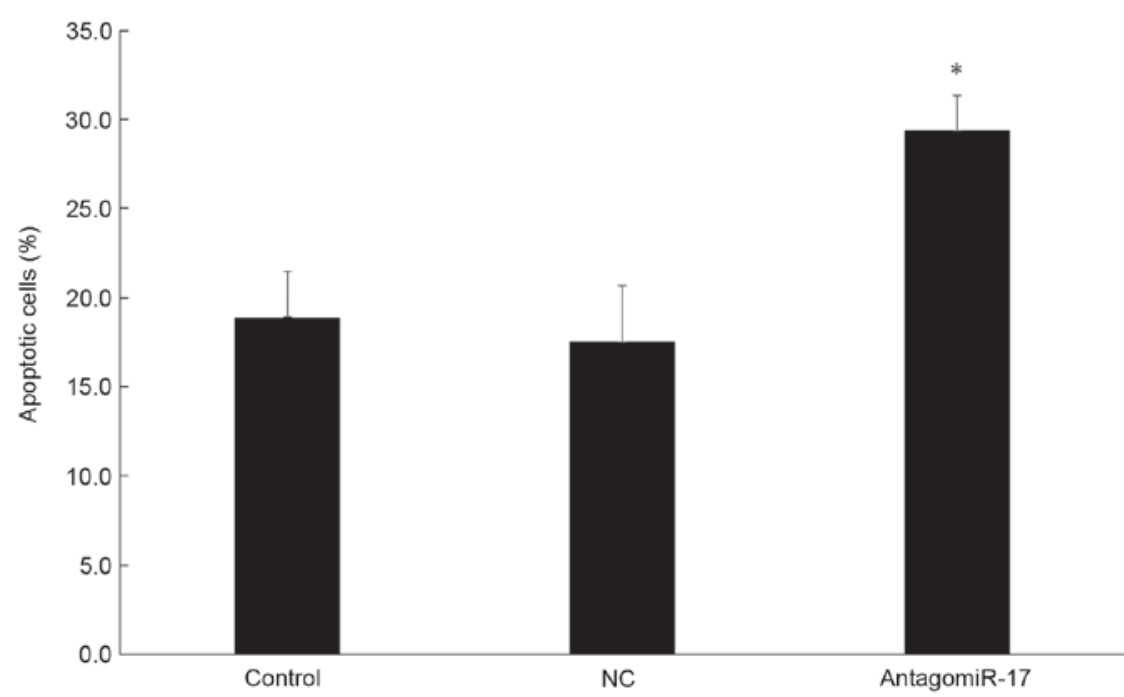

Figure 3. Effect of miR-17 on the apoptosis of endometrial cells. (A) Cell apoptosis rates were analyzed by flow cytometry. (B) Percentage of apoptotic cells following downregulation of miR-17. "P<0.05 vs. control group. miR-17, microRNA-17; NC, negative control; PI, propidium iodide.

A

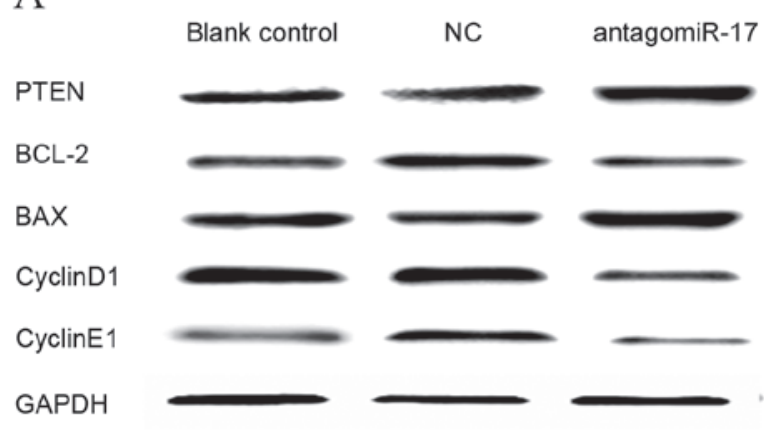

B

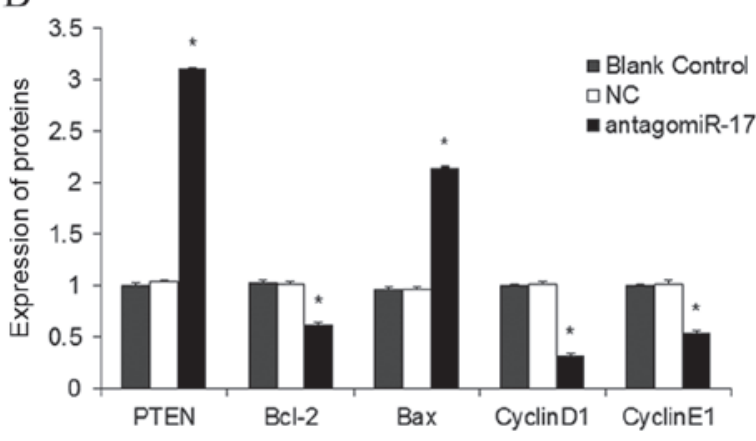

Figure 4. Effect of miR-17 on the expression of PTEN, cell cycle-related and apoptosis-related proteins. (A) Expression of PTEN, Bcl-2, Bax, cyclins D1 and E1 proteins was evaluated by western blot analysis. (B) Quantification of western blot results. The protein levels were normalized to GADPH. * $<0.05$ vs. NC. miR-17, microRNA-17; NC, negative control.

These results indicate that miR-17 may bind to the 3'-UTR of PTEN, thus regulating its expression.

\section{Discussion}

miRs are gene regulation factors involved in numerous pathophysiological processes, including the proliferation, invasion and metastasis of tumor cells, hypertension, diabetes and atherosclerosis $(21,22)$. Studies are currently investigating the functions of an increasing number of miRs. However, the expression and acting mechanisms of miRs in AM remain unclear. It has been reported that certain miRs, including miR-191, miR-142-3p, miR-29c and miR-210, serve important roles in endometriosis $(23,24)$. The pathogenesis of AM and endometriosis are similar as they both result from the invasion and metastasis of endometrial cells (25-27). Therefore, it was speculated that miRs may also be involved in AM.

miR-17 is a tumor-related miRNA that serves important roles in the proliferation, invasion and metastasis of tumor cells (28). In AM, the invasion and metastastic abilities of 
A

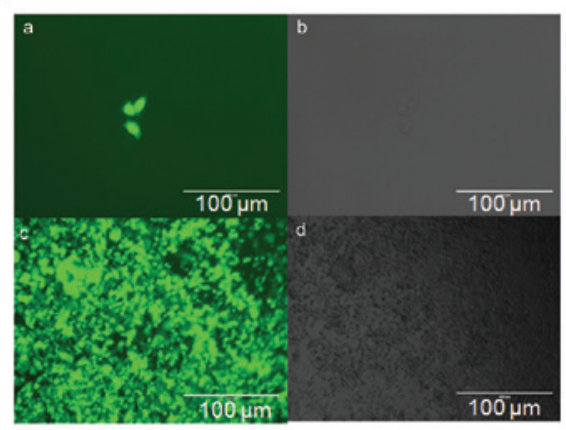

B

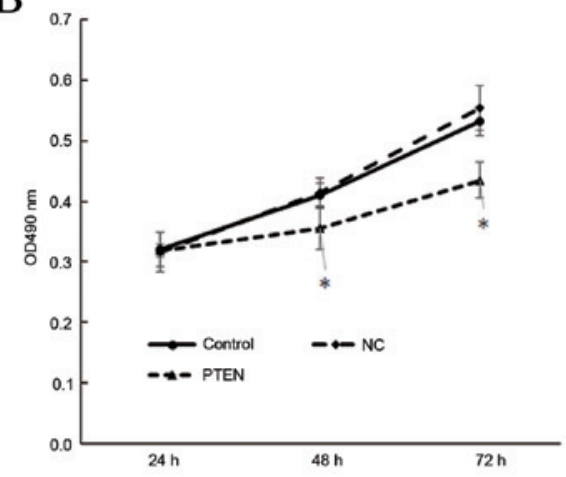

C

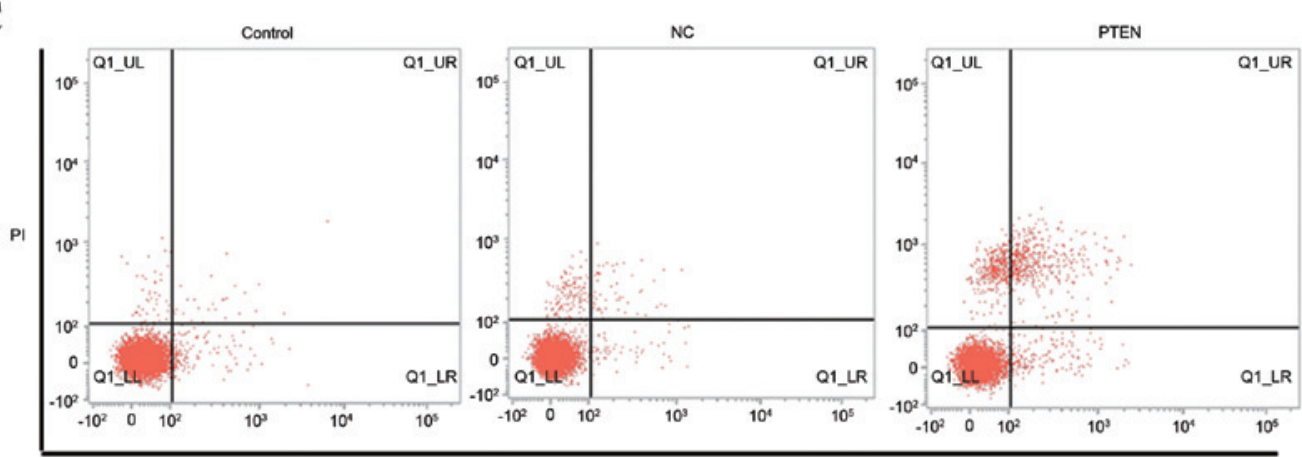

Annexin V

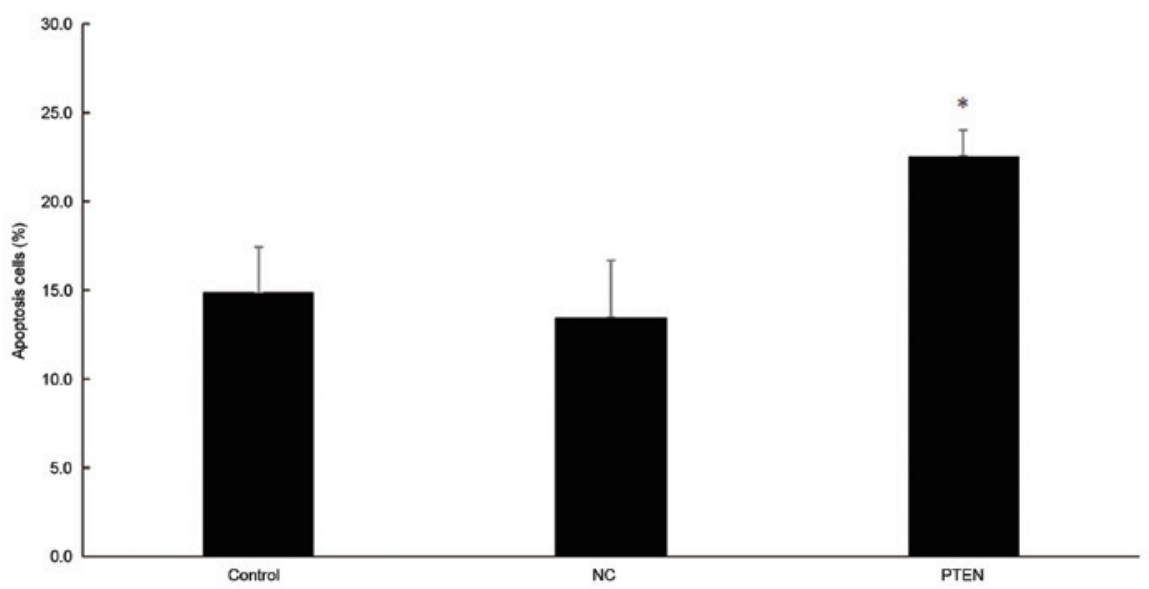

Figure 5. Effect of PTEN overexpression on endometrial cells. (A) Images of endometrial cells screened by G418 for PTEN overexpression: (a) Monoclonal cell group with GFP fluorescence; (b) bright light image of the same cell group under the same view as (a); (c) monoclonal cell group with GFP fluorescence following expending culture; (d) bright light image of the same cell group under the same view as (c). Scale bar=100 $\mu \mathrm{m}$. Magnification, x100. (B) Effect of PTEN overexpression on the viability of endometrial cells, as determined by MTT assay. (C) Effect of PTEN on the apoptosis rate of endometrial cells, detected by flow cytometry. "P<0.05 vs. control group. GFP, green fluorescent protein; NC, negative control; PTEN, phosphatase and tensin homolog.

endometrial cells are enhanced, which is an important cause of the disease (29). To the best of our knowledge, the present study was the first to investigate whether miR-17 is involved in altering the biological functions of endometrial cells. The results indicated that miR-17 expression in the endometrial tissues of AM patients was significantly increased compared with control patients. It was predicted by online bioinformatics analysis that miR-17 may be involved in the regulation of PTEN gene expression. Previous results indicated that the PTEN gene is closely related to the proliferation, apoptosis, invasion and metastasis of cells (30). Therefore, it was speculated that the upregulation of miR-17 in AM endometrial cells resulted in the downregulation of PTEN, thus influencing the biological properties of endometrial cells. Results from RT-qPCR and western blot analysis

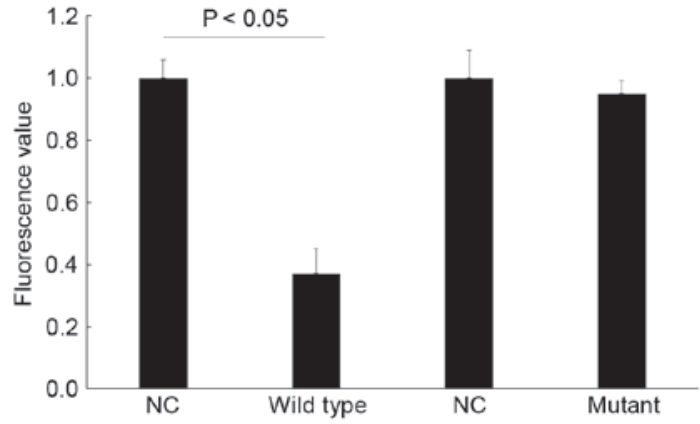

Figure 6. Identification of the microRNA-17 target gene by dual-luciferase reporter assay. Cells were transfected with a reporter plasmid containing wild type or mutant 3'-untranslated region of the PTEN gene. NC, negative control. 
indicated that the expression of PTEN mRNA and protein in AM endometrial tissues was significantly lower compared with the control group. To further study the molecular mechanism of miR-17, ESCs were cultured in vitro and transfected with antagomiR-17 using the liposome method. ESC viability was measured using an MTT assay, and it was found that miR-17 could promote cell viability. Results from western blotting indicated that the expression of PTEN protein increased when miR-17 expression was downregulated, whereas the expression of Bcl-2 decreased and that of Bax increased. Results from flow cytometry indicated that miR-17 may inhibit endometrial cell apoptosis. In addition, a PTEN stably expressed endometrial cell line was constructed in vitro and functional changes were investigated. The results indicated that when PTEN protein was overexpressed, cell viability significantly decreased and cell apoptosis significantly increased. In order to verify the direct binding of miR-17 and PTEN mRNA, a dual-luciferase reporter assay was performed and indicated that miR-17 could bind to the 3'-UTR seed sequence of PTEN mRNA and regulate its expression.

In conclusion, the expression of miR-17 was increased in the endometrial tissues of AM. miR-17 expression may regulate the expression of PTEN and affect the expression of apoptosis- and cell cycle-related proteins. This suggests that miR-17 may serve a role in promoting cell viability, invasion and metastasis in the development of AM.

\section{Acknowledgements}

The present study was supported by the National Natural Science Foundation of China (grant no. 81270658) and the Science and Technology Project of Shenzhen (grant no. 201402065).

\section{References}

1. Lee JS, Hong GY, Park BJ and Kim TE: Ultrasound-guided high-intensity focused ultrasound treatment for uterine fibroid \& adenomyosis: A single center experience from the Republic of Korea. Ultrason Sonochem 27: 682-687, 2015.

2. Streuli I, Santulli P, Chouzenoux S, Chapron C and Batteux F: Activation of the MAPK/ERK Cell-signaling pathway in uterine smooth muscle cells of women with adenomyosis. Reprod Sci 22: $1549-1560,2015$.

3. Struble J, Reid S and Bedaiwy MA: Adenomyosis: A clinical review of a challenging gynecologic condition. J Minim Invasive Gynecol 23: 164-185, 2016.

4. Wang Y, Qu Y and Song W: Genetic variation in COX-2 -1195 and the risk of endometriosis and adenomyosis. Clin Exp Obstet Gynecol 42: 168-172, 2015.

5. Fawzy M and Mesbah Y: Comparison of dienogest versus triptorelin acetate in premenopausal women with adenomyosis: A prospective clinical trial. Arch Gynecol Obstet 292: 1267-1271, 2015.

6. Zhihong N, Yun F, Pinggui Z, Sulian Z and Zhang A: Cytokine profiling in the eutopic endometrium of adenomyosis during the implantation window after ovarian stimulation. Reprod Sci 23: 124-133, 2016.

7. Graziano A, Lo Monte G, Piva I, Caserta D, Karner M, Engl B and Marci R: Diagnostic findings in adenomyosis: A pictorial review on the major concerns. Eur Rev Med Pharmacol Sci 19: 1146-1154, 2015.

8. Barbieri RL: Primary gonadotropin-releasing hormone agonist therapy for suspected endometriosis: A nonsurgical approach to the diagnosis and treatment of chronic pelvic pain. Am J Manag Care 3: 285-290, 1997.

9. Jia W, Wu Y, Zhang Q, Gao GE, Zhang C and Xiang Y: Expression profile of circulating microRNAs as a promising fingerprint for cervical cancer diagnosis and monitoring. Mol Clin Oncol 3: 851-858, 2015.
10. Juzėnas S, Saltenienè V, Kupcinskas J, Link A, Kiudelis G, Jonaitis L, Jarmalaite S, Kupcinskas L, Malfertheiner P and Skieceviciene J: Analysis of 'deregulated microRNAs and their target genes in gastric cancer. PLoS One 10: e0132327, 2015.

11. Jiang XI, Luo Y, Zhao S, Chen Q, Jiang C, Dai Y, Chen Y and Cao Z: Clinical significance and expression of microRNA in diabetic patients with erectile dysfunction. Exp Ther Med 10: 213-218, 2015.

12. Mogilyansky E and Rigoutsos I: The miR-17/92 cluster: A comprehensive update on its genomics, genetics, functions and increasingly important and numerous roles in health and disease. Cell Death Differ 20: 1603-1614, 2013.

13. Tan W, Li Y, Lim SG and Tan TM: MiR-106b-25/miR-17-92 clusters: Polycistrons with oncogenic roles in hepatocellular carcinoma. World J Gastroenterol 20: 5962-5972, 2014.

14. Ferrari F, Arrigoni F, Miccoli A, Mascaretti S, Fascetti E, Mascaretti G, Barile A and Masciocchi C: Effectiveness of magnetic resonance-guided focused ultrasound surgery (MRgFUS) in the uterine adenomyosis treatment: Technical approach and MRI evaluation. Radiol Med 121: 153-161, 2016.

15. Quattrochi B, Gulvady A, Driscoll DR, Sano M, Klimstra DS, Turner CE and Lewis BC: MicroRNAs of the mir-17 92 cluster regulate multiple aspects of pancreatic tumor development and progression. Oncotarget 8: 35902-35918, 2017.

16. Mei J, Zhu XY, Jin LP, Duan ZL, Li DJ and Li MQ: Estrogen promotes the survival of human secretory phase endometrial stromal cells via CXCL12/CXCR4 up-regulation-mediated autophagy inhibition. Hum Reprod 30: 1677-1689, 2015.

17. Monteiro MB, Santos-Bezerra DP, Thieme K, Passarelli M, Machado UF, Lin CJ and Corrêa-Giannella ML: Optimization of total RNA isolation from human urinary sediment. Clin Chim Acta 462: 158-161, 2016.

18. Livak KJ and Schmittgen TD: Analysis of relative gene expression data using real-time quantitative PCR and the 2(-Delta Delta C(T)) Method. Methods 25: 402-408, 2001.

19. Wang J, Zhou J, Gowtham YK, Harcum SW and Husson SM: Antibody purification from $\mathrm{CHO}$ cell supernatant using new multimodal membranes. Biotechnol Prog 33: 658-665, 2017.

20. Rasoulianboroujeni M, Kupgan G, Moghadam F, Tahriri M, Boughdachi A, Khoshkenar P, Ambrose JJ, Kiaie N, Vashaee D, Ramsey JD and Tayebi L: Development of a DNA-liposome complex for gene delivery applications. Mater Sci Eng C Mater Biol Appl 75: 191-197, 2017.

21. Varshney J and Subramanian S: MicroRNAs as potential target in human bone and soft tissue sarcoma therapeutics. Front Mol Biosci 2: 31, 2015.

22. Liz J and Esteller M: lncRNAs and microRNAs with a role in cancer development. Biochim Biophys Acta 1859: 169-176, 2016.

23. Ohlsson Teague EM, Van der Hoek KH, Van der Hoek MB, Perry N, Wagaarachchi P, Robertson SA, Print CG and Hull LM: MicroRNA-regulated pathways associated with endometriosis. Mol Endocrinol 23: 265-275, 2009.

24. Tian X, Xu L and Wang P: MiR-191 inhibits TNF- $\alpha$ induced apoptosis of ovarian endometriosis and endometrioid carcinoma cells by targeting DAPK1. Int J Clin Exp Pathol 8: 4933-4942, 2015.

25. Hawkins SM, Creighton CJ, Han DY, Zariff A, Anderson ML, Gunaratne PH and Matzuk MM: Functional microRNA involved in endometriosis. Mol Endocrinol 25: 821-832, 2011.

26. Teague EM, Print CG and Hull ML: The role of microRNAs in endometriosis and associated reproductive conditions. Hum Reprod Update 16: 142-165, 2010.

27. Braza-Boïls A, Marí-Alexandre J, Gilabert J, Sánchez-Izquierdo D, España F, Estellés A and Gilabert-Estellés J: MicroRNA expression profile in endometriosis: Its relation to angiogenesis and fibrinolytic factors. Hum Reprod 29: 978-988, 2014.

28. Fuziwara CS and Kimura ET: Insights into regulation of the miR-17-92 cluster of miRNAs in cancer. Front Med (Lausanne) 2: 64, 2015.

29. Zhou S, Yi T, Liu R, Bian C, Qi X, He X, Wang K, Li J, Zhao X, Huang $\mathrm{C}$ and Wei Y: Proteomics identification of annexin A2 as a key mediator in the metastasis and proangiogenesis of endometrial cells in human adenomyosis. Mol Cell Proteomics 11: M112. 017988, 2012.

30. Costa HA, Leitner MG, Sos ML, Mavrantoni A, Rychkova A, Johnson JR, Newton BW, Yee MC, De La Vega FM, Ford JM, et al: Discovery and functional characterization of a neomorphic PTEN mutation. Proc Natl Acad Sci USA 112: 13976-13981, 2015. 